\title{
Application of Fuzzy Reasoning Spiking Neural P Systems to Fault Diagnosis
}

\author{
T. Wang, G. Zhang, H. Rong, M.J. Pérez-Jiménez
}

\author{
Tao Wang, Gexiang Zhang*, Haina Rong \\ School of Electrical Engineering, Southwest Jiaotong University \\ Chengdu, 610031, China \\ wangtaocdu@gmail.com, zhgxdylan@126.com \\ ronghaina@126.com \\ *Corresponding author: wangtaocdu@gmail.com \\ Mario J. Pérez-Jiménez \\ Research Group on Natural Computing \\ Department of Computer Science and Artificial Intelligence \\ University of Sevilla, Sevilla, 41012, Spain \\ marper@us.es
}

\begin{abstract}
This paper discusses the application of fuzzy reasoning spiking neural $\mathrm{P}$ systems with trapezoidal fuzzy numbers (tFRSN P systems) to fault diagnosis of power systems, where a matrix-based fuzzy reasoning algorithm based on the dynamic firing mechanism of neurons is used to develop the inference ability of tFRSN P systems from classical reasoning to fuzzy reasoning. Some case studies show the effectiveness of the presented method. We also briefly draw comparisons between the presented method and several main fault diagnosis approaches from the perspectives of knowledge representation and inference process.

Keywords: fuzzy reasoning spiking neural $\mathrm{P}$ system with trapezoidal fuzzy number, fuzzy reasoning, fault diagnosis, trapezoidal fuzzy number, linguistic term.
\end{abstract}

\section{Introduction}

Membrane computing, introduced by Gh. Păun in [1], is an attractive research field of computer science aiming at abstracting computing models, called membrane systems or $\mathrm{P}$ systems, from the structures and functioning of living cells, as well as from the way the cells are organized in tissues or higher order structures. In recent years, much attention is paid to the spiking neural P systems (SN P systems, for short), an important class of P systems introduced in [2] and investigated in a series of papers (see [3]- [12]), which can be described as a directed graph. An SN P system is a kind of distributed and parallel computing model inspired by the neurophysiological behavior of neurons sending electrical impulses (spikes) along axons from presynaptic neurons to postsynaptic neurons. The features of SN P systems, such as inherent parallelism, understandability, dynamics, synchronization/asychronization, non-linearity and nondeterminism [3], [4], are suitable for solving various engineering problems.

Until now, only a few investigations have focused on the use of SN P systems to solve engineering problems. In [3], a fuzzy reasoning spiking neural $\mathrm{P}$ system with real numbers (rFRSN P system) was presented to perform diagnosis knowledge representation and reasoning. In [13], an rFRSN P system was used for fault diagnosis of power systems and three examples were used to verify its effectiveness. The studies in [3,13], dealing with the fault diagnosis problem, used certainty factors and truth degree values, which are described by real numbers obtained from the frequency of occurrences in historical data, It is known how difficult it is to obtain and process real-time statistical data from power network data and the knowledge of dispatchers and experts in electrical power systems as they usually contain linguistic terms with 
some degree of uncertainty. So, using various models, including rFRSN P systems to solve fault diagnosis problems with a certain degree of uncertainty, represents a way to tackle this difficult problem.

This paper discusses the extended version of rFRSN P systems, i.e., fuzzy reasoning spiking neural P systems with trapezoidal fuzzy numbers (tFRSN P systems), and its application to fault diagnosis of power systems. To adapt tFRSN P systems to solve fault diagnosis problems, a matrix-based fuzzy reasoning algorithm (MBFRA) is used inspired by the dynamic firing mechanism of neurons. Given initial pulse values of all input neurons of a tFRSN P system, MBFRA can perform fuzzy inference to obtain the pulse values contained in other neurons and export reasoning results represented by trapezoidal fuzzy numbers. To make MBFRA suitable for multiple faults diagnosis of power systems, a defuzzification method is applied for processing the reasoning results in order to obtain crisp numbers corresponding to them. Some case studies show the effectiveness of the presented method. We also briefly draw comparisons between tFRSN P systems and several other fault diagnosis approaches.

The remainder of this paper is organized as follows. Section 2 introduces concepts and notations used in this work. Section 3 provides the definition of tFRSN P systems and MBFRA. Section 4 discusses the application of tFRSN P systems to fault diagnosis of power systems. Discussions on several fault diagnosis methods are made in Section 5. Conclusions are finally drawn in section 6 .

\section{Preliminaries}

A trapezoidal fuzzy number can be characterized as a 4-tuple of real numbers $\tilde{T}_{f}=(a, b, c, d), a<$ $b<c<d$, shown in Fig. 1, where $a$ and $d$ represents the left hand and right hand width of the trapezoidal distribution, $(b, c)$ denotes the interval in which the membership value is equal to 1 and $H_{\tilde{T}_{f}}(x)$ represents the membership function of $\tilde{T}_{f}$ defined as follows [14]:

$$
H_{\tilde{T}_{f}}(x)=\left\{\begin{array}{cl}
0, & x \leq a \\
\frac{x-a}{b-a}, & a<x \leq b \\
1, & b<x \leq c \\
\frac{d-x}{d-c}, & c<x \leq d \\
0, & x>d
\end{array}\right.
$$

Let $\tilde{A}$ and $\tilde{B}$ be two trapezoidal fuzzy numbers, $\tilde{A}=\left(a_{1}, b_{1}, c_{1}, d_{1}\right)$ and $\tilde{B}=\left(a_{2}, b_{2}, c_{2}, d_{2}\right)$. The arithmetic operations of $\tilde{A}$ and $\tilde{B}$ are defined as follows [15]:

1. Addition $\oplus: \tilde{A} \oplus \tilde{B}=\left(a_{1}, b_{1}, c_{1}, d_{1}\right) \oplus\left(a_{2}, b_{2}, c_{2}, d_{2}\right)=\left(a_{1}+a_{2}, b_{1}+b_{2}, c_{1}+c_{2}, d_{1}+d_{2}\right)$;

2. Subtraction $\ominus: \tilde{A} \ominus \tilde{B}=\left(a_{1}, b_{1}, c_{1}, d_{1}\right) \ominus\left(a_{2}, b_{2}, c_{2}, d_{2}\right)=\left(a_{1}-a_{2}, b_{1}-b_{2}, c_{1}-c_{2}, d_{1}-d_{2}\right)$;

3. Multiplication $\otimes: \tilde{A} \otimes \tilde{B}=\left(a_{1}, b_{1}, c_{1}, d_{1}\right) \otimes\left(a_{2}, b_{2}, c_{2}, d_{2}\right)=\left(a_{1} \times a_{2}, b_{1} \times b_{2}, c_{1} \times c_{2}, d_{1} \times d_{2}\right)$;

4. Division $\oslash: \tilde{A} \oslash \tilde{B}=\left(a_{1}, b_{1}, c_{1}, d_{1}\right) \oslash\left(a_{2}, b_{2}, c_{2}, d_{2}\right)=\left(a_{1} / a_{2}, b_{1} / b_{2}, c_{1} / c_{2}, d_{1} / d_{2}\right)$.

We define four logic operations, where $\tilde{A}$ and $\tilde{B}$ are trapezoidal fuzzy numbers, and $a, b$ are real numbers:

1. Minimum operator $\wedge: a \wedge b=\min (a, b)$;

2. Maximum operator $\vee: a \vee b=\max (a, b)$; 


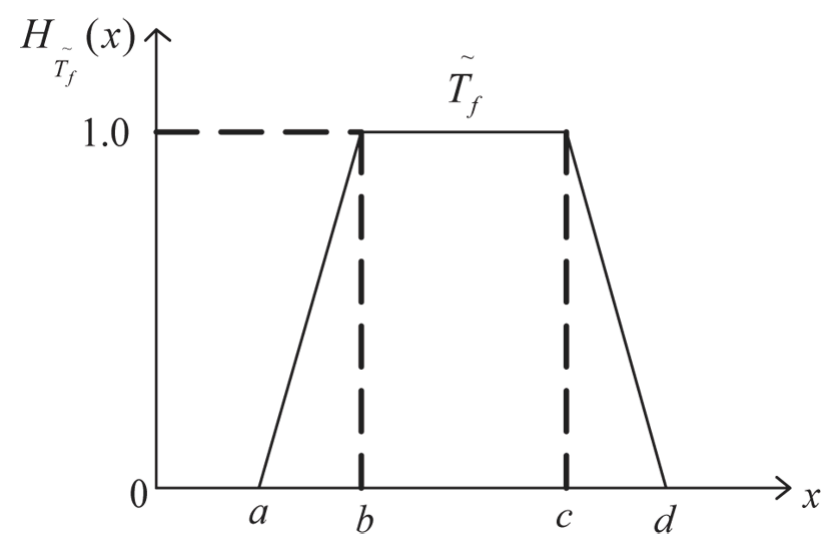

Figure 1: A trapezoidal fuzzy number.

3. and $₫: \tilde{A} \oplus \tilde{B}=\left(a_{1}, b_{1}, c_{1}, d_{1}\right) \oplus\left(a_{2}, b_{2}, c_{2}, d_{2}\right)=\left(\left(a_{1} \wedge a_{2}\right),\left(b_{1} \wedge b_{2}\right),\left(c_{1} \wedge c_{2}\right),\left(d_{1} \wedge d_{2}\right)\right)$;

4. or $(\nabla): \tilde{A} \otimes \tilde{B}=\left(a_{1}, b_{1}, c_{1}, d_{1}\right) \otimes\left(a_{2}, b_{2}, c_{2}, d_{2}\right)=\left(\left(a_{1} \vee a_{2}\right),\left(b_{1} \vee b_{2}\right),\left(c_{1} \vee c_{2}\right),\left(d_{1} \vee d_{2}\right)\right)$.

We define a scalar multiplication operation as follows:

Scalar Multiplication: $x \cdot \tilde{A}=x \cdot\left(a_{1}, b_{1}, c_{1}, d_{1}\right)=\left(x \cdot a_{1}, x \cdot b_{1}, x \cdot c_{1}, x \cdot d_{1}\right)$, where $A$ is a trapezoidal fuzzy number and $x$ is a real number:

The defuzzification method in [16] is chosen to obtain a crisp number $t_{f}$ associated with a trapezoidal fuzzy number $\tilde{T}_{f}$, it is shown in (2), where $e$ and $g$ are the extreme values of the whole fuzzy set range. In this study, $e$ and $g$ are equal to 0 and 1 , respectively.

$$
t_{f}=\frac{(d-e)+(c-e)}{((d-e)+(c-e))-((a-g)+(b-g))}
$$

\section{$3 \quad$ tFRSN P systems}

A tFRSN $\mathrm{P}$ system of $m \geq 1$ is a construct $\Pi=\left(O, \sigma_{1}, \ldots, \sigma_{m}\right.$, syn, in, out $)$, where:

(1) $O=\{a\}$ is a singleton alphabet ( $a$ is called spike);

(2) $\sigma_{1}, \ldots, \sigma_{m}$ are neurons, of the form $\sigma_{i}=\left(\theta_{i}, c_{i}, r_{i}\right), 1 \leq i \leq m$, where:

(a) $\theta_{i}$ is a trapezoidal fuzzy number in $[0,1]$ representing the potential value of spikes (i.e. value of electrical impulses) contained in neuron $\sigma_{i}$;

(b) $c_{i}$ is a trapezoidal fuzzy number in $[0,1]$ representing the fuzzy truth value corresponding to neuron $\sigma_{i}$;

(c) $r_{i}$ represents a firing (spiking) rule contained in neuron $\sigma_{i}$ with the form $E / a^{\theta} \rightarrow a^{\beta}$, where $E$ is the firing condition and its form will be specified below, $\theta$ and $\beta$ are trapezoidal fuzzy numbers in $[0,1]$.

(3) syn $\subseteq\{1,2, \ldots, m\} \times\{1,2, \ldots, m\}$ with $i \neq j$ for all $(i, j) \in$ syn, $1 \leq i, j \leq m$, is a directed graph of synapses between the linked neurons;

(4) in, out $\subseteq\{1,2, \ldots, m\}$ indicate the input neuron set and the output neuron set of $\Pi$, respectively. 
In tFRSN P systems, the definition of neurons and pulse values can be extended. Specifically, in tFRSN P systems, the neurons are extended to four types, i.e., proposition neurons and three kinds of rule neurons: general, and and or, and the pulse value contained in each neuron is no longer the number of spikes represented by a real value, but a trapezoidal fuzzy number in $[0,1]$, which can be interpreted as the potential value of spikes contained in neuron $\sigma_{i}$. It is worth pointing out that the number of spikes in each neuron is determined by the problem to be solved and the pulse value contained in each neuron is different. If neuron $\sigma_{i}$ contains no spike, then $\theta_{i}=0$; otherwise, if neuron $\sigma_{i}$ contains only one spike, then $\theta_{i}$ equals to the pulse value of this spike; in any other case, $\theta_{i}$ equals to the result of a operation on all pulse values received from its presynaptic neurons. For different types of neurons, the operations for pulse values are different. For proposition neurons and and rule neurons, they use operation $(\wedge$ to handle all the pulse values received from their presynaptic neurons while or rule neurons use operation $(\nabla)$, where symbols $(\wedge$ and $(\nabla$ represent the and and or operators of trapezoidal fuzzy numbers, respectively. The firing condition $E=a^{s}$ means that the spiking rule, $E / a^{\theta} \rightarrow a^{\beta}$, contained in neuron $\sigma_{i}$, can be applied if and only if neuron $\sigma_{i}$ contains at least $s$ spikes, otherwise, the firing rule cannot be applied. More details about tFRSN P systems can be found to the preliminary work [9].

Fuzzy production rules consist of five types:

Type 1: $R_{i}\left(c_{i}\right): p_{j}\left(\theta_{j}\right) \rightarrow p_{k}\left(\theta_{k}\right) ; \theta_{k}=\theta_{j} \otimes c_{i}$.

Type 2: $R_{i}\left(c_{i}\right): p_{1}\left(\theta_{1}\right) \bowtie \ldots\left(p_{k-1}\left(\theta_{k-1}\right) \rightarrow p_{k}\left(\theta_{k}\right) ; \theta_{k}=\left(\theta_{1} \bowtie \ldots \bowtie \theta_{k-1}\right) \otimes c_{i}\right.$.

Type 3: $R_{i}\left(c_{i}\right): p_{1}\left(\theta_{1}\right) \rightarrow p_{2}\left(\theta_{2}\right) \bowtie \ldots \bowtie p_{k}\left(\theta_{k}\right) ; \theta_{2}=\ldots \theta_{k}=\theta_{1} \otimes c_{i}$.

Type 4: $R_{i}\left(c_{i}\right): p_{1}\left(\theta_{1}\right) \otimes \ldots \bigotimes p_{k-1}\left(\theta_{k-1}\right) \rightarrow p_{k}\left(\theta_{k}\right) ; \theta_{k}=\left(\theta_{1} \otimes \ldots\left(\theta_{k-1}\right) \otimes c_{i}\right.$.

Type 5: $R_{i}\left(c_{i}\right): p_{1}\left(\theta_{1}\right) \rightarrow p_{2}\left(\theta_{2}\right) \otimes \ldots \bigotimes p_{k}\left(\theta_{k}\right)$.

where $R_{i}$ represents the $i$ th fuzzy production rule; $c_{i}$ is the certainty factor of rule $R_{i} ; p_{i}$ is a proposition appearing in the antecedent or consequence part of a rule, $1 \leq i \leq k$ ( $k$ is the number of propositions in a rule-based system); $p_{j}$ in Type 1 represents the $j$ th proposition, $1 \leq j \leq k-1$; $\theta_{i}$ represents the fuzzy truth value corresponding to the $i$ th proposition [15]. $c_{i}$ and $\theta_{i}$ are trapezoidal fuzzy numbers defined in the universe of discourse $[0,1]$. The causality between a fault on a faulty section in a power system and the status information about protective relays and circuit breakers $(\mathrm{CBs})$ of this section can be described by the aforementioned fuzzy production rules. A simplified transmission network shown in Fig. 2 is used to illustrate the notations in a fuzzy production rule. According to the protection principle, if there is fault on transmission $L$, then its main protective relays, i.e., $M L R_{1}$ and $M L R_{2}$ and their corresponding CBs, i.e., $C B_{1}$ and $C B_{2}$, will operate to protect $L$, which can be backward described by a fuzzy production rule: $R(1,1,1,1): M L R_{1}$ operates $(0.975,0.98,1,1) \oplus M L R_{2}$ operates $(0.975,0.98,1,1)(\uparrow$ $C B_{1}$ trips $(0.975,0.98,1,1)\left(C B_{2}\right.$ trips $(0.975,0.98,1,1) \rightarrow L$ has a fault. The certainty factor of this rule is $(1,1,1,1)$ which represents the contribution of this rule to the final diagnosis result. In this rule, there are four propositions $M L R_{1}$ operates, $M L R_{2}$ operates, $C B_{1}$ trips and $C B_{2}$ trips, and they have an equal fuzzy truth value $(0.975,0.98,1,1)$ representing the contributions of the propositions to the result $L$ has a fault.

Because rules Type 5 are unsuitable for diagnosis, they are not further described in Section 3. tFRSN P system models for rules Type 1 to Type 4 are shown in Fig. 3.

To adapt tFRSN $\mathrm{P}$ systems to solve fault diagnosis problems, we describe MBFRA in the following description.

Given initial truth values of propositions corresponding to all input neurons in an tFRSN $\mathrm{P}$ system, MBFRA can perform fuzzy reasoning to obtain the fuzzy truth values of other neurons with unknown pulse values and output reasoning results. Let us assume that the tFRSN P system contains $l$ proposition neurons and $n$ rule neurons, each of which may be general, and or or rule neurons, $m=l+n$, where $m$ is the number of all the neurons in this system. 


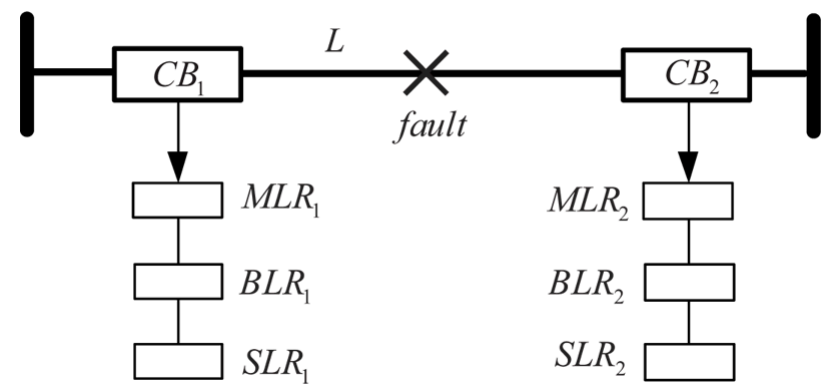

Figure 2: A simplified transmission network.

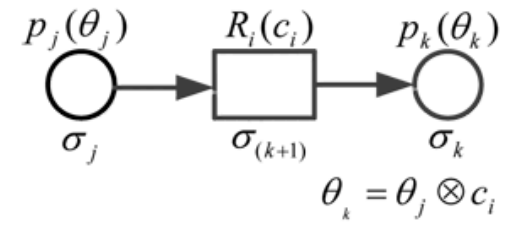

(a)

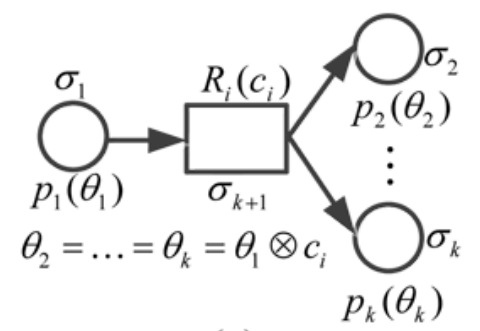

(c)

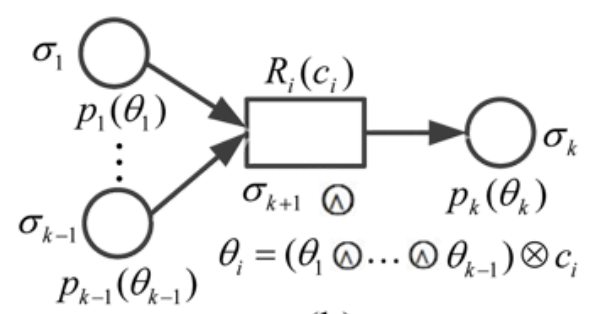

(b)

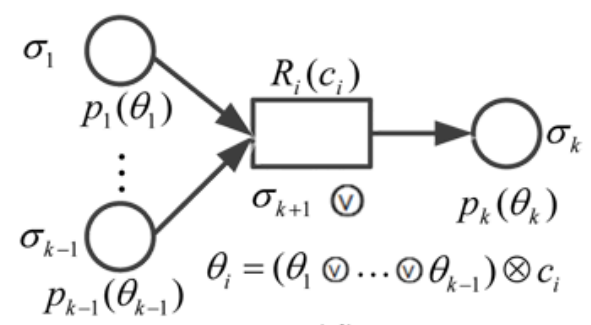

(d)

Figure 3: tFRSN P system models for fuzzy production rules. (a) Type 1; (b) Type 2; (c) Type 3; (d) Type 4.

In order to clearly present the reasoning algorithm, we first introduce some parameter vectors and matrices as follows.

1) $\boldsymbol{\theta}=\left(\theta_{1}, \theta_{2}, \ldots, \theta_{l}\right)^{T}$ is a fuzzy truth value vector of the $l$ proposition neurons, where $\theta_{i}$ represents the pulse value contained in the $i$ th proposition neuron, $1 \leq i \leq l$, and is expressed by a trapezoidal fuzzy number in $[0,1]$. If there is not any spike contained in a proposition neuron, its pulse value is "unknown" or $(0,0,0,0)$.

2) $\boldsymbol{\delta}=\left(\delta_{1}, \delta_{2}, \ldots, \delta_{n}\right)^{T}$ is a fuzzy truth value vector of the rule neurons, where $\delta_{j}$ represents the pulse value contained in the $j$ th rule neuron, $1 \leq j \leq n$, and it is expressed by a trapezoidal fuzzy number $[0,1]$. If there is not any spike contained in a rule neuron, its pulse value is "unknown" or $(0,0,0,0)$.

3) $C=\operatorname{diag}\left(c_{1}, c_{2}, \ldots, c_{n}\right)$ is a diagonal matrix, where $c_{j}$ is the certainty factor of the $j$ th fuzzy production rule, $1 \leq j \leq n$, and it is expressed by a trapezoidal fuzzy number.

4) $\boldsymbol{D}_{1}=\left(d_{i j}\right)_{l \times n}$ is a synaptic matrix representing the direct connection between proposition neurons and general rule neurons. If there is a directed arc (synapse) from the proposition neuron $\sigma_{i}$ to the general rule neuron $\sigma_{j}$, then $d_{i j}=1$, otherwise, $d_{i j}=0$. 
5) $\boldsymbol{D}_{2}=\left(d_{i j}\right)_{l \times n}$ is a synaptic matrix representing the direct connection between proposition neurons and and rule neurons. If there is a directed arc (synapse) from the proposition neuron $\sigma_{i}$ to the and rule neuron $\sigma_{j}$, then $d_{i j}=1$, otherwise, $d_{i j}=0$.

6) $\boldsymbol{D}_{3}=\left(d_{i j}\right)_{l \times n}$ is a synaptic matrix representing the direct connection between proposition neurons and or rule neurons. If there is a directed arc (synapse) from the proposition neuron $\sigma_{i}$ to the or rule neuron $\sigma_{j}$, then $d_{i j}=1$, otherwise, $d_{i j}=0$.

7) $\boldsymbol{E}=\left(e_{j i}\right)_{n \times l}$ is a synaptic matrix representing the direct connection between rule neurons and proposition rule neurons. If there is a directed arc (synapse) from the rule neuron $\sigma_{j}$ to the proposition neuron $\sigma_{i}$, then $e_{j i}=1$, otherwise, $e_{j i}=0$.

Subsequently, we introduce some multiplication operations as follows.

1) $\odot: ~ C \boldsymbol{\delta} \odot=\left(c_{1} \otimes \delta_{1}, c_{2} \otimes \delta_{2}, \ldots, c_{n} \otimes \delta_{n}\right)^{T} ; \boldsymbol{D}^{T} \odot \boldsymbol{\theta}=\left(\bar{d}_{1}, \bar{d}_{2}, \ldots, \bar{d}_{n}\right)^{T}$, where $\bar{d}_{j}$ $=d_{1 j} \theta_{1}+d_{2 j} \theta_{2}+\ldots+d_{l j} \theta_{l}, j=1,2, \ldots, n$.

2) $\odot: \boldsymbol{D}^{T} \odot \boldsymbol{\theta}=\left(\bar{d}_{1}, \bar{d}_{2}, \ldots, \bar{d}_{n}\right)^{T}$, where $\bar{d}_{j}=d_{1 j} \theta_{1} \bowtie d_{2 j} \theta_{2}\left(\ldots \Uparrow d_{l j} \theta_{l}, j=1,2, \ldots, n\right.$.

3) $\circledast: \boldsymbol{E}^{T} \circledast \boldsymbol{\delta}=\left(\overline{e_{1}}, \overline{e_{2}}, \ldots, \overline{e_{l}}\right)^{T}$, where $\overline{e_{i}}=e_{1 i} \delta_{1}$ ( $e_{2 i} \delta_{2}$ ( $\ldots$ ( $) e_{n i} \delta_{n}, i=1,2, \ldots, l$.

Next, we list the pseudocode of MBFRA.

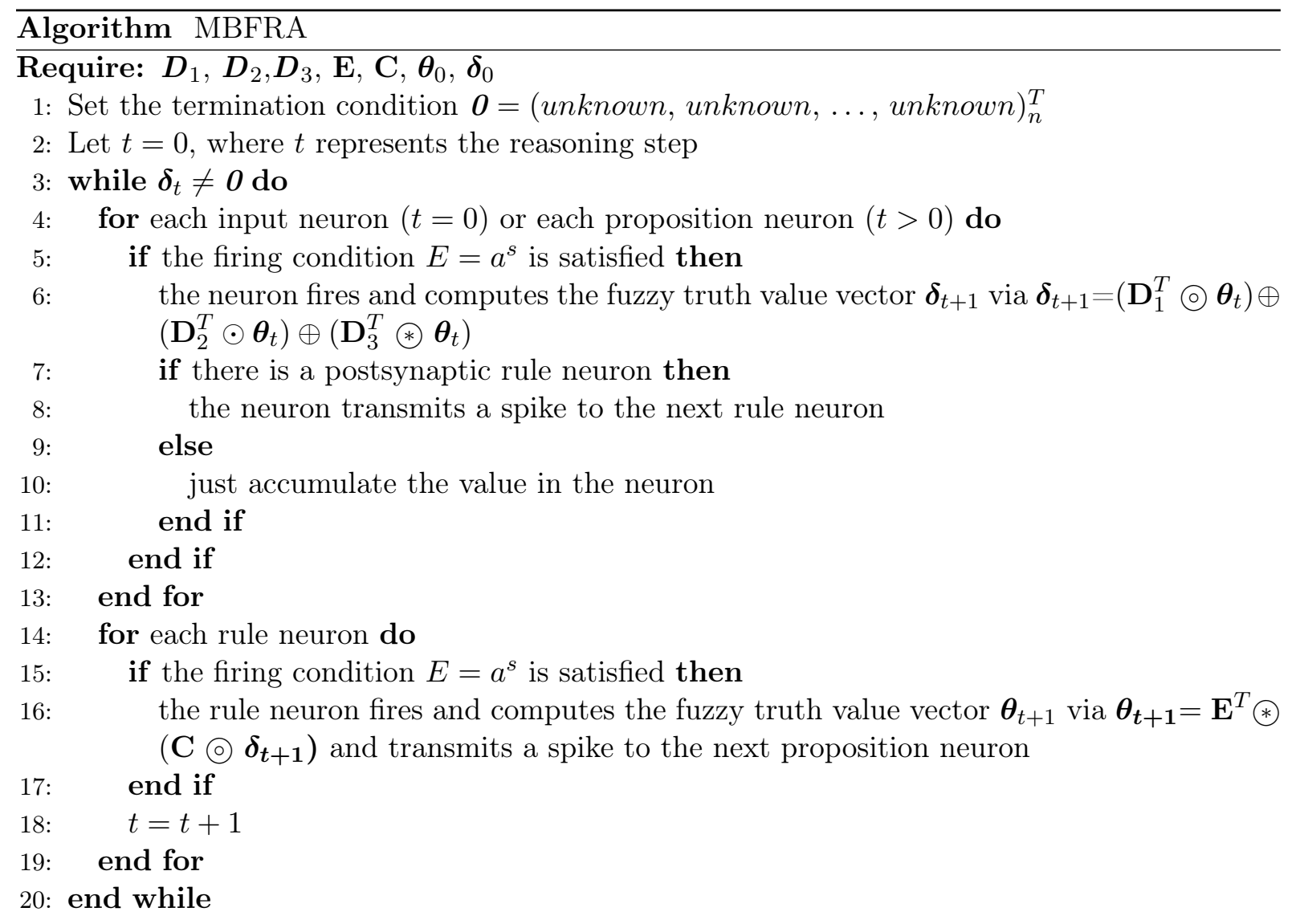

Ensure: $\boldsymbol{\theta}_{t}$, which represents the final states of pulse values contained in proposition neurons.

\section{Application Examples and Results}

In this section, a power system with 14-buses, chosen from [17] and as shown in Fig. 4, is applied as an example to describe how to use tFRSN P systems with MBFRA to solve a fault diagnosis problem. The system contains 34 system sections, including 14 buses and 20 transmission lines. The buses are marked as $B_{p q}$ and the transmission lines are represented 


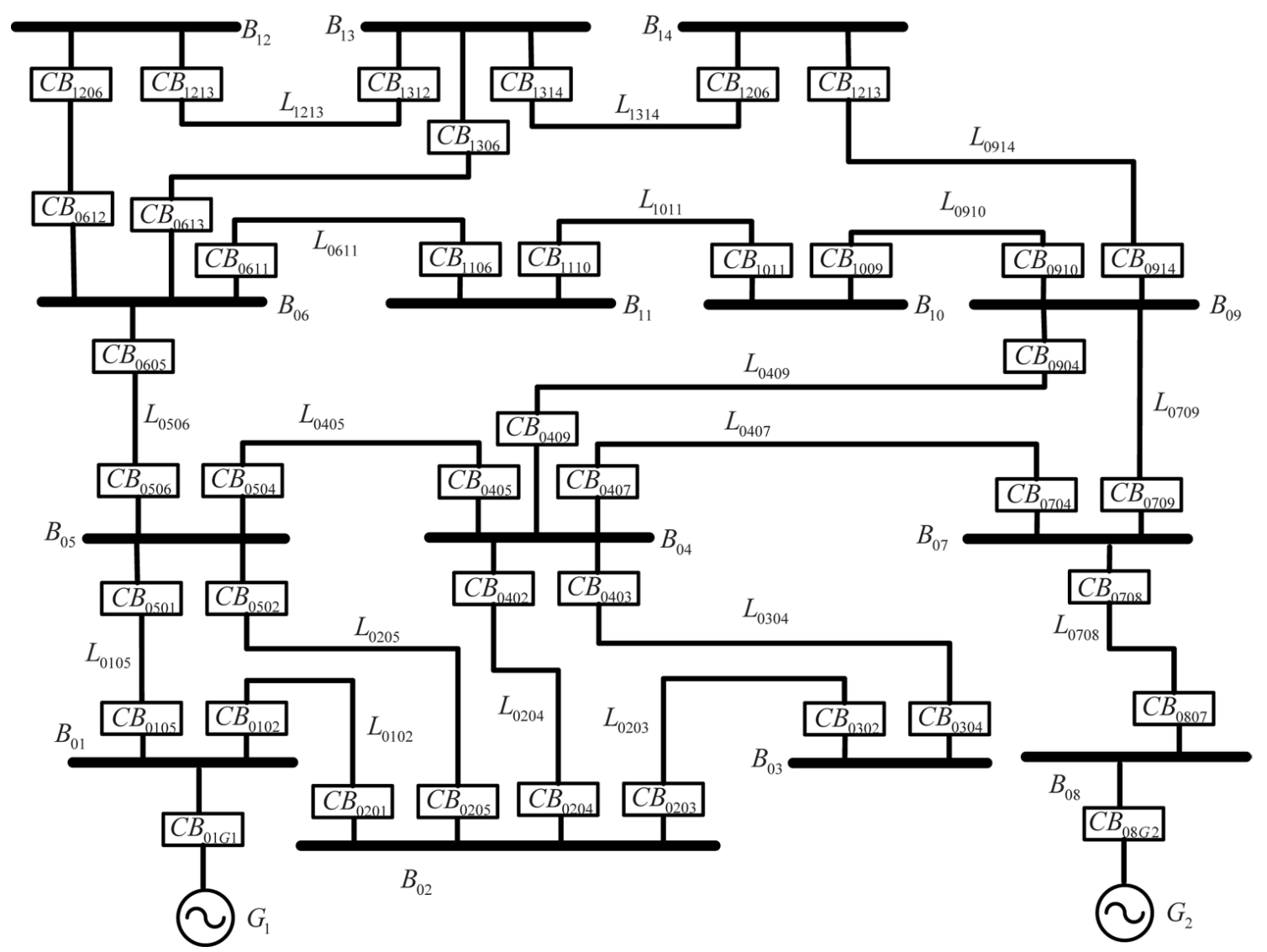

Figure 4: The power system with 14 buses.

as $L_{p q u v}$, where $0 \leq p, q, u, v \leq 9$. The protection system of the 14 -bus system contains 174 protective devices consisting of 40 circuit breakers (CBs), 40 main transmission line relays, 40 first backup transmission line relays, 40 second backup transmission line relays and 14 bus relays. A local part, which is composed of a transmission line $L_{1314}$, its adjoining two buses, $B_{13}$ and $B_{14}$, and its adjoining three transmission lines, $L_{1213}, L_{0613}$ and $L_{0914}$, of the protection system is given to describe its structure and symbols of protection devices. The local system is shown in Fig. 5. The operational rules of the protective devices are descried as follows [17].

The main transmission line relay $M L R_{1314}$ protects the entire line $L_{1314}$ and it will operate to trip its associated circuit breaker (CB), i.e., $C B_{1314}$, to clear a fault on the line $L_{1314}$. The bus relay $B R_{13}$ protects the bus $B_{13}$ and it will operate to trip the three $\mathrm{CBs}$, i.e., $C B_{1312}, C B_{1306}$ and $C B_{1314}$, if there is a fault on the bus $B_{13}$. The first backup transmission line relay $B L R_{1314}$ is a local backup of the relay $M L R_{1314}$ and has the same protection zone as $M L R_{1314}$. The relay $B L R_{1314}$ will operate to trip $C B_{1314}$ to clear a fault if the fault clearance by the relay $M L R_{1314}$ fails. Secondary backup transmission line relays $S L R_{1213}$ and $S L R_{0613}$ are the remote backups of the relays $M L R_{1314}$ and $B L R_{1314}$. They will operate to trip their corresponding CBs, i.e., $C B_{1213}$ and $C B_{0613}$, respectively, to clear a fault if the fault clearance by both $M L R_{1314}$ and $B L R_{1314}$ fails. The relays $S L R_{1213}$ and $S L R_{0613}$ are also two remote backups of the relay $B R_{13}$ and they will operate to trip CBs, i.e., $C B_{1213}$ and $C B_{0613}$, respectively, to clear a fault if the fault clearance by the relay $B R_{13}$ fails. The functions of the four relays, $M L R_{1413}, B L R_{1413}$, $S L R_{0914}$ and $B R_{14}$, and three CBs, $C B_{1413}, C B_{1409}$ and $C B_{0914}$, in the process of protecting the line $L_{1314}$ and the bus $B_{14}$ are similar and the protection systems for other sections in this 14-bus power system have the same protection rules, so it is not necessary to repeatedly describe 


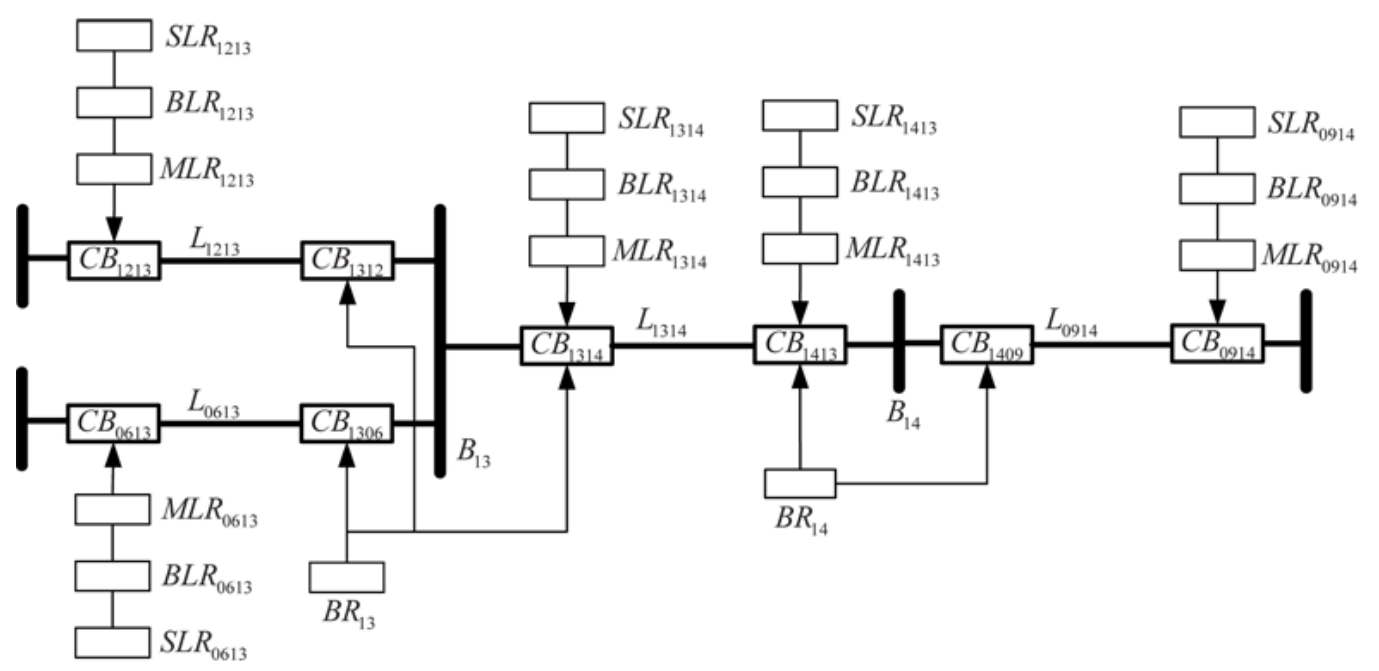

Figure 5: A local part of the protection system of the 14-bus power system.

their operation rules.

The protection rules described above show that when a fault occurs on a certain section of a power system, protection devices will reach certain statuses to protect the section. Meanwhile, the relay trip signals and CBs status signals, used as inputs of fault diagnosis models of sections, can be obtained from remote terminal units (RTUs) of supervisory control and data acquisition (SCADA) systems. The diagnostic strategy in this study is to build one tFRSN P system diagnosis model for each candidate fault section of a power system and each model performs MBFRA by using SCADA data, i.e., relay trip signals and CBs status signals, to get a trapezoidal fuzzy number which represents the fault confidence level of this section. In a single fault case, the section with the highest fault confidence level is the faulty section. In multiple faults cases, several sections with fault confidence levels which are greater than a threshold, which is set as real number 0.5 in this study, are regarded as faulty sections. Thus, to obtain real numbers for easily comparing the fault confidence levels with the threshold, a defuzzification method shown in (2) is used to process the reasoning results represented by trapezoidal fuzzy numbers. In addition, the fault confidence levels of faulty sections in multiple faults cases are ranked from high to low to help operators to decide a repair order of the sections.

Fig. 6 and Fig. 7 show the tFRSN P system diagnosis models for $L_{1314}$ and $B_{13}$, respectively. It is worth noting that there are several assistant arcs (synapsises) with different arrow endings in the figures. For illustration purposes, we take arcs, from $\sigma_{2}$ to $\sigma_{25}$ and from $\sigma_{2}$ to $\sigma_{26}$, as examples. The meanings of the two arcs are that if $C B_{1314}$ opens, the operation of its corresponding second backup protective devices, including relays $\left(S L R_{0613}\right.$ and $\left.S L R_{1213}\right)$ and CBs $\left(C B_{0613}\right.$ and $\left.C B_{1213}\right)$, is invalid and then the values of these relays and CBs are set as $(0,0,0,0)$; otherwise, the operation of the second backup protective devices is valid. In what follows we take transmission line $L_{1314}$ as an example to show the fuzzy reasoning process of MBFRA based on tFRSN P systems.

Case 1: A single fault. Transmission line $L_{1314}$ has a fault.

Operated relays: $M L R_{1314}, M L R_{1413}, B L R_{1314}$. Tripped CBs: $C B_{1314}, C B_{1413}$.

A tFRSN P system for $L_{1314}$ is $\Pi_{1}$ and its corresponding tFRSN P system diagnosis model is shown in Fig. 6.

$$
\Pi_{1}=\left(O, \sigma_{1}, \sigma_{2}, \ldots, \sigma_{36}, \text { syn, in }, \text { out }\right)
$$




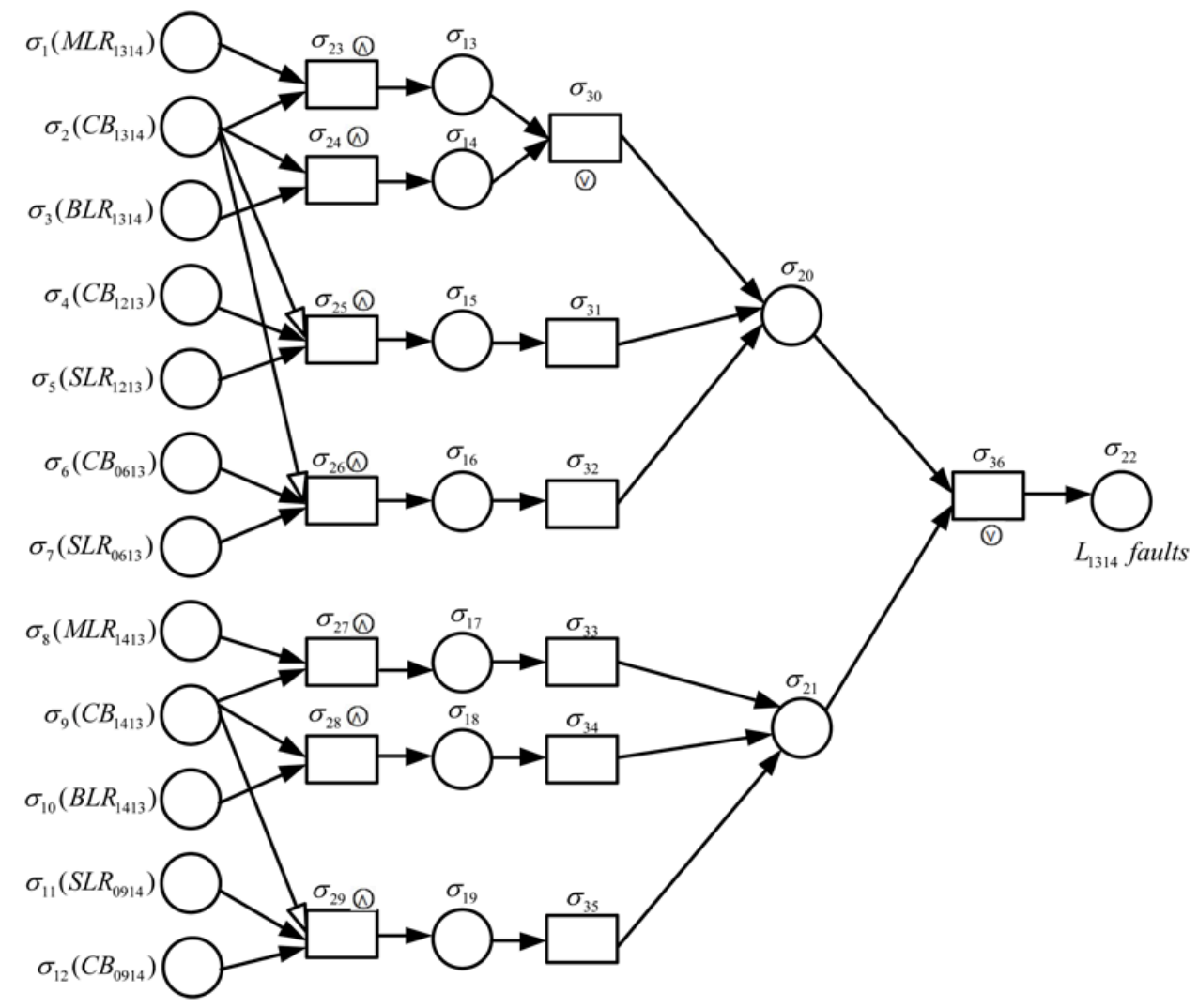

Figure 6: Fault diagnosis model of transmission line $L_{1314}$ based on a tFRSN P system.

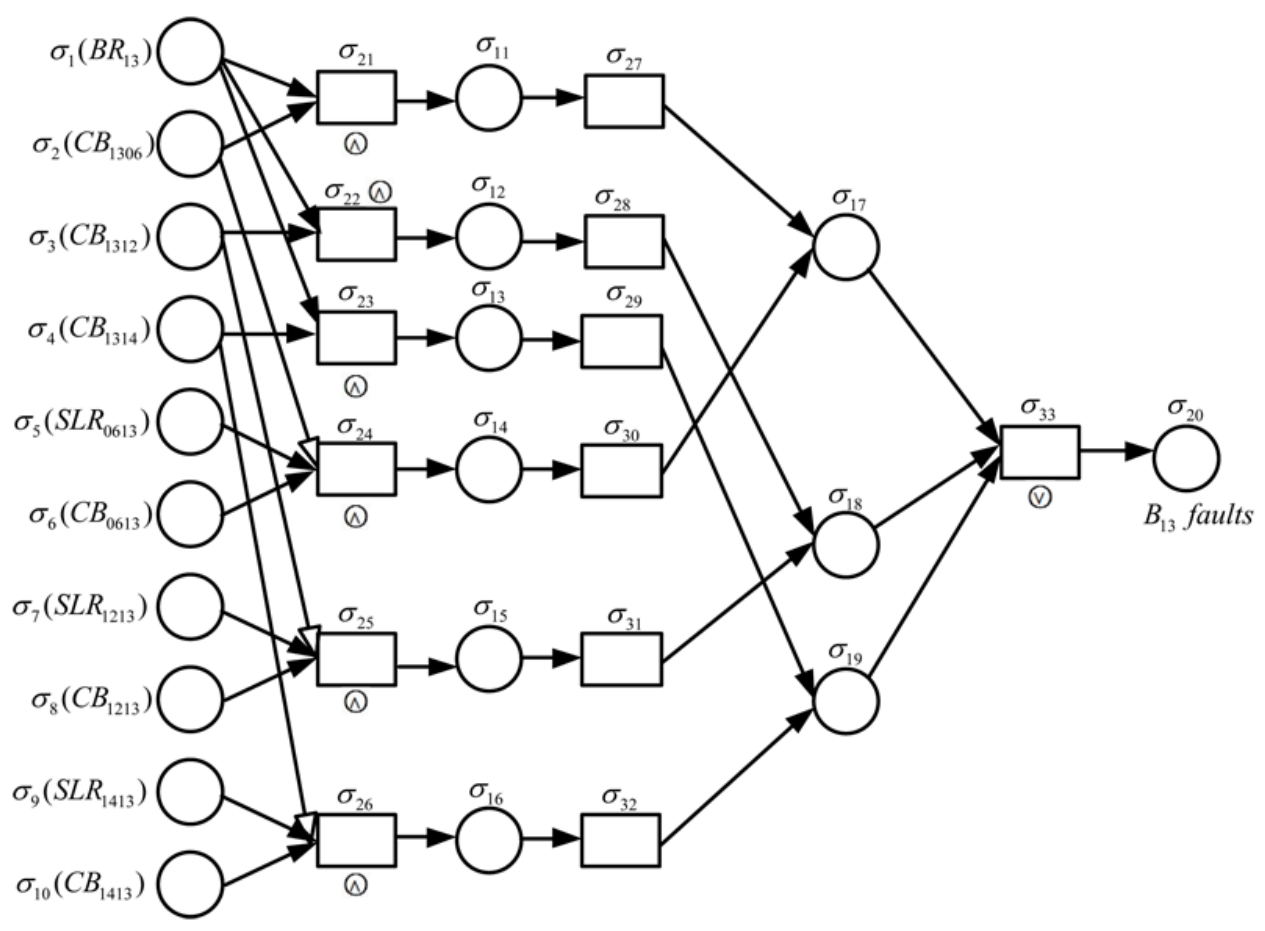

Figure 7: Fault diagnosis model of bus $B_{13}$ based on a tFRSN P system. 
Table 1: Linguistic terms and their corresponding trapezoidal fuzzy numbers

\begin{tabular}{ll}
\hline Linguistic Terms & Trapezoidal Fuzzy Numbers \\
\hline absolutely-false (AF) & $(0,0,0,0)$ \\
very-low (VL) & $(0,0,0.02,0.07)$ \\
low (L) & $(0.04,0.1,0.18,0.23)$ \\
medium-low (ML) & $(0.17,0.22,0.36,0.42)$ \\
medium (M) & $(0.32,0.41,0.58,0.65)$ \\
medium-high (MH) & $(0.58,0.63,0.80,0.86)$ \\
high (H) & $(0.72,0.78,0.92,0.97)$ \\
very-high $(\mathrm{VH})$ & $(0.975,0.98,1,1)$ \\
absolutely-high $(\mathrm{AH})$ & $(1,1,1,1)$ \\
\hline
\end{tabular}

where

1) $O=\{a\}$ is the singleton alphabet ( $a$ is called spike).

2) $\sigma_{1}, \ldots, \sigma_{22}$ are proposition neurons corresponding to the propositions with fuzzy truth values $\theta_{1}, \ldots, \theta_{22}$; that is, $l=22$.

3) $\sigma_{23}, \ldots, \sigma_{36}$ are rule neurons, where $\sigma_{23}, \ldots, \sigma_{29}$ are and rule neurons, $\sigma_{30}$ and $\sigma_{36}$ are or rule neurons and $\sigma_{31}, \ldots, \sigma_{35}$ are general rule neurons; that is, $n=14$.

4) $\operatorname{syn}=\{(1,23),(2,23),(2,24),(2,25),(2,26),(3,24),(4,25),(5,25),(6,26),(7,26)$, $(8,27),(9,27),(9,28),(9,29),(10,28),(11,29),(12,29),(13,30),(14,30),(15,31),(16,32),(17,33)$, $(18,34),(19,35),(20,36),(21,36),(23,13),(24,14),(25,15),(26,16),(27,17),(28,18),(29,19),(30$, $20),(31,20),(32,20),(33,21),(34,21),(35,21),(36,22)\}$.

5) in $=\left\{\sigma_{1}, \sigma_{2}, \ldots, \sigma_{12}\right\}$, out $=\left\{\sigma_{22}\right\}$.

The knowledge of dispatchers in power systems may contain linguistic terms and the statuses of devices may have a certain degree of uncertainty. Table 1 shows an example of linguistic terms and their corresponding trapezoidal fuzzy numbers. In the tFRSN $\mathrm{P}$ system $\Pi_{1}$, input neurons $\sigma_{1}, \ldots, \sigma_{12}$ are assigned as the empirical values $V H, V H, H, A F, A F, A F, A F, V H, V H, L$, $A F, A F$, respectively. Certainty factors corresponding to rule neurons $\sigma_{23}, \ldots, \sigma_{36}$ are given values $V H, V H, V H, H, V H, V H, H, V H, V H, V H, V H, V H, V H, V H$, respectively.

According to Table 1 , we obtain the trapezoidal fuzzy numbers $\boldsymbol{\theta}_{\mathbf{0}}$ and $\boldsymbol{\delta}_{\mathbf{0}}$. In order to succinctly describe the matrices, let us denote $\boldsymbol{O}_{r}=\left(x_{1}, \ldots, x_{r}\right)^{T}$, where $x_{i}=(0,0,0,0), 1 \leq$ $i \leq r$.

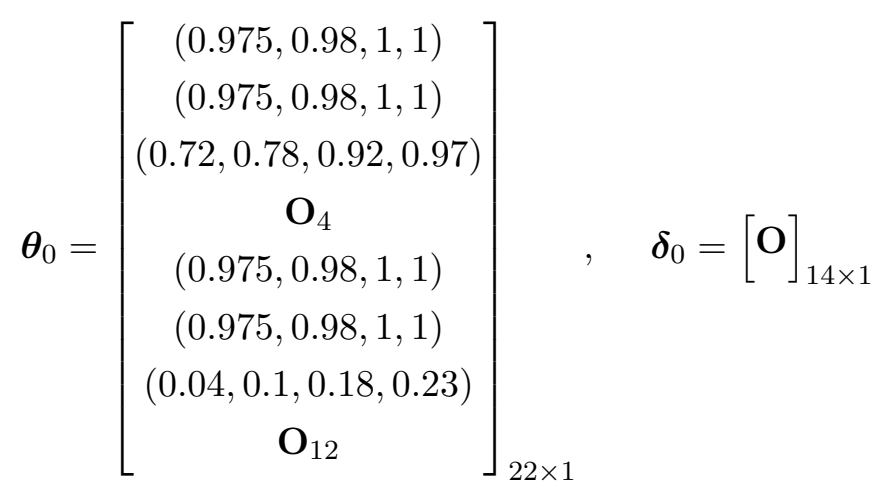

When $t=0$, we get the results 


$$
\boldsymbol{\delta}_{1}=\left[\begin{array}{c}
(0.9506,0.9604,1,1) \\
(0.702,0.7644,0.92,0.97) \\
\mathbf{O}_{2} \\
(0.9506,0.9604,1,1) \\
(0.39,0.098,0.18,0.23) \\
\mathbf{O}_{8}
\end{array}\right]_{14 \times 1}, \quad \boldsymbol{\theta}_{1}=\left[\begin{array}{c}
\mathbf{O}_{12} \\
(0.9268,0.9412,1,1) \\
(0.6845,0.7491,0.92,0.97) \\
\mathbf{O}_{2} \\
(0.9268,0.9412,1,1) \\
\mathbf{O}_{4}
\end{array}\right]_{22 \times 1}
$$

When $t=1$, we obtain the results

$$
\boldsymbol{\delta}_{2}=\left[\begin{array}{c}
\mathbf{O}_{7} \\
(0.9268,0.9412,1,1) \\
\mathbf{O}_{2} \\
(0.9268,0.9412,1,1) \\
\mathbf{O}_{2}
\end{array}\right]_{14 \times 1}, \quad \boldsymbol{\theta}_{2}=\left[\begin{array}{c}
\mathbf{O}_{19} \\
(0.9268,0.9412,1,1) \\
(0.9268,0.9412,1,1) \\
\mathbf{O}_{1}
\end{array}\right]_{22 \times 1}
$$

When $t=2$, we have the results

$$
\boldsymbol{\delta}_{3}=\left[\begin{array}{c}
\mathbf{O}_{13} \\
(0.9268,0.9412,1,1)
\end{array}\right]_{14 \times 1}, \quad \boldsymbol{\theta}_{3}=\left[\begin{array}{c}
\mathbf{O}_{21} \\
(0.9036,0.9224,1,1)
\end{array}\right]_{22 \times 1}
$$

When $t=3$, we get the results

$$
\boldsymbol{\delta}_{4}=[\mathbf{O}]_{14 \times 1} .
$$

Thus, the termination condition is satisfied and the reasoning process ends. We obtain the reasoning results, i.e., the fuzzy truth value $(0.9036,0.9224,1,1)$ of the output neuron $\sigma_{22}$. The transmission line $L_{1314}$ is a faulty section with a confidence level $(0.9036,0.9224,1,1)$.

tFRSN P systems and MBFRA are also suitable for multiple faults diagnosis problems in power systems. In what follows we take an example of the power system in Fig. 4 to show the effectiveness of the method in diagnosing multiple faults.

Case 2: Multiple faults. Transmission line $L_{1314}$ and bus $B_{13}$ have faults.

Operated relays: $M L R_{1314}, M L R_{1413}, S L R_{0613}, S L R_{1213}$. Tripped CBs: $C B_{1314}, C B_{1413}$, $C B_{0613}, C B_{1213}$.

According to the SCADA data, four candidate fault sections, i.e., $L_{1314}, B_{13}, L_{0613}$ and $L_{1213}$, are selected. The tFRSN P systems of the four sections are established to perform MBFRA, respectively. After the fuzzy reasoning, fault confidence levels, $(0.9036,0.9224,1,1),(0.6673$, $0.7341,0.92,0.97),(0.2165,0.299,0.623,0.7849)$ and $(0.2165,0.299,0.623,0.7849)$, represented by trapezoidal fuzzy numbers of sections $L_{1314}, B_{13}, L_{0613}$ and $L_{1213}$ are obtained. According to (2), we obtain their corresponding real numbers, i.e., 0.92, 0.7595, 0.475 and 0.475 . Thus, there are two faulty sections, i.e., $L_{1314}$ and $B_{13}$. The results are summarized in Table 2 .

The logic analysis about Case 2 is described as follows. In this case, information of protective relay $B R_{13}$ is not observed and $C B_{1312}$ and $C B_{1306}$ fail to trip. For transmission line $L_{1314}$, its main transmission relays, $M L R_{1314}$ and $M L R_{1413}$, operate to trip their corresponding $\mathrm{CBs}$, 
Table 2: Relay trip signals and CBs status signals observed, and diagnosis results

\begin{tabular}{ccccc}
\hline $\begin{array}{c}\text { Candidate } \\
\text { fault section }\end{array}$ & $\begin{array}{c}\text { Confidence } \\
\text { level }\end{array}$ & $\begin{array}{c}\text { Corresponding } \\
\text { real number }\end{array}$ & Ranking & $\begin{array}{c}\text { Fault } \\
\text { section }\end{array}$ \\
\hline$L_{1314}$ & $(0.9036,0.9224,1,1)$ & 0.92 & 1 & Yes \\
$B_{13}$ & $(0.6673,0.7341,0.92,0.97)$ & 0.7595 & 2 & Yes \\
$L_{0613}$ & $(0.2165,0.299,0.623,0.7849)$ & 0.475 & - & No \\
$L_{1213}$ & $(0.2165,0.299,0.623,0.7849)$ & 0.475 & - & No \\
\hline
\end{tabular}

$C B_{1314}$ and $C B_{1413}$, to clear a fault. So it is a faulty section. Although main protective relay $B R_{13}$ of bus $B_{13}$ fails to clear a fault, its remote backup protective relays, $S L R_{0613}$ and $S L R_{1213}$, operate to trip their corresponding CBs, $C B_{0613}$ and $C B_{1213}$, to clear this fault. So $B_{13}$ is a faulty section. For transmission lines $L_{0613}$ and $L_{1213}$, only their single-ended remote backup protective relays $S L R_{0613}$ and $S L R_{1213}$ operate to trip their corresponding CBs, $C B_{0613}$ and $C B_{1213}$, respectively. Actually, $S L R_{0613}$ and $S L R_{1213}$ and their CBs act as remote backup protections of $B_{13}$. So, $L_{0613}$ and $L_{1213}$ are not faulty sections. Therefore, according to the logic analysis and Table 2, we can know that the presented method can obtain correct results in multiple fault situations.

\section{Discussions}

A tFRSN P system is a novel graphical model for representing fuzzy knowledge and information. This study employs it to diagnose the faults of power systems. The fault diagnosis ability of a method is usually associated with the knowledge availability and the reasoning process. Thus, in what follows, we make a comparison between tFRSN P systems and several fault diagnosis approaches regarding the aspects of knowledge representation and inference process.

(1) Expert systems (ESs). Both ES and the fault diagnosis method based on tFRSN P systems (FDM-tFRSNP) can make full use of experts' knowledge. The differences are: an ES needs long response time and the maintenance of its knowledge base is difficult [18]; FDMtFRSNP possesses parallel reasoning ability and adopt graphical knowledge representation and reasoning, which can avoid the main limitation of ES.

(2) Fuzzy set theory (FST). FST is an effective way to represent uncertain information but the definition of membership function is a hard job [18]. The FST-based method and FDM-tFRSNP both possess the ability to deal with uncertain information of protective devices. In addition, linguistic terms used in both methods make them closer to the human thinking compared with the methods using crisp numbers. The main differences between them are that FDM-tFRSNP has a fast reasoning speed and the matrix reasoning process is easier to describe diagnostic process as well as its programming.

(3) Artificial neural networks (ANNs). ANNs can be regarded as opaque black boxes and can be easily used. The main problems of ANNs lie in the difficult acquisition of a complete sample set and a tedious training process needing extra time consumption. In addition, premature convergence is also a problem. FDM-tFRSNP neither needs a training process with a set of comprehensive training data nor has a premature convergence problem [13]. Besides, FDMtFRSNP can intuitively represent the relationships between faults and operations of protection devices. This feature is very helpful for operators to analyze and summarize failure processes.

(4) Fuzzy Petri nets (FPNs). Both FPNs and tFRSN P systems have graphical knowledge representation and parallel computing ability. However, the mechanism of tFRSN P systems is originated from neurophysiological behavior of neurons or/and living cells. Thus, the working principle of different types of neurons or/and cells may provide new inspirations for extending SN 
P systems (or tFRSN P systems), which can increase the ways of knowledge representation and reasoning to solve new problems in power systems [4]. In addition, tFRSN P systems with trapezoidal fuzzy numbers have three rule neurons types, i.e., general, and and or, and one proposition neuron type, while FPNs only contain same places and transition types. Thus, different types of neurons make FDM-tFRSNP have better flexibility and trapezoidal fuzzy numbers (linguistic terms) make tFRSN P systems more understandable to operators of power systems.

\section{Conclusions}

In this study, tFRSN P systems and a matrix-based fuzzy reasoning algorithm, MBFRA, for fault diagnosis are discussed to extend the application area of SN P systems in fault diagnosis of power systems. MBFRA is based on the dynamic firing mechanism of neurons. Given initial pulse values of all input neurons of a tFRSN P system, MBFRA can obtain the pulse values of other neurons by performing fuzzy reasoning. To make MBFRA suitable for fault diagnosis in power systems, a defuzzification method is employed to treat reasoning results represented by trapezoidal fuzzy numbers. Application examples show that tFRSN P systems with MBFRA is effective in diagnosing faulty sections of power systems. Besides, a comparison between tFRSN $\mathrm{P}$ systems and different fault diagnosis approaches is made.

The aim of this study is to construct a tFRSN P system diagnosis model for each candidate fault section. The scale of each diagnosis model depends on protective devices connections of the candidate fault section rather than the scale of power systems. Thus, the presented method can be used for large-scale power systems. This study focuses on the effectiveness and correctness of the fault diagnosis method and the results of application examples are obtained by manual computation. To test the speed, convergence and accuracy of MBFRA and to explore automatical generation of tFRSN P systems in diagnosing faulty sections in power systems, our future work will simulate them on MATLAB, P-Lingua or MeCoSim [19]- [21]. Moreover, how to verify and realize the parallelism of tFRSN P systems and MBFRA on hardware such as FPGA and CUDA is also our further task.

\section{Acknowledgment}

This work is supported by the National Natural Science Foundation of China (61170016, 61373047, 61170030), the Program for New Century Excellent Talents in University (NCET-110715) and SWJTU supported project (SWJTU12CX008).

\section{Bibliography}

[1] Păun, G. (2000); Computing with Membranes, J Comput. Syst. Sci., ISSN 0022-0000, 61(1): 108-143.

[2] Ionescu, M.; Păun, G.; Yokomori T. (2006); Spiking Neural P Systems, Fund. Inform., ISSN 0169-2968, 71(2-3): 279-308.

[3] Peng, H.; Wang, J.; Pérez-Jiménez, M.J.; Wang, H.; Shao, J.; Wang, T. (2013); Fuzzy Reasoning Spiking Neural P System for Fault Diagnosis, Inform. Sciences, ISSN 0020-0255, 235(20): 106-116.

[4] Wang, J.; Shi, P.; Peng, H.; Pérez-Jiménez, M.J.; Wang, T. (2013); Weighted Fuzzy Spiking Neural P Systems, IEEE Trans. on Fuzzy Syst., ISSN 1063-6706, 21(2): 209-220. 
[5] Păun, G.; Pérez-Jiménez, M.J.; Rozenberg, G. (2006); Spike Trains in Spiking Neural P Systems, Int. J. Found. Comput. S., ISSN 0129-0541, 17(4): 975-1002.

[6] Cavaliere, M.; Ibarra, O.H.; Păun, G.; Egecioglu, O.; Ionescu, M.; Woodworth, S. (2009); Asynchronous Spiking Neural P Systems, Theor. Comput. Sci., ISSN 0304-3975, 410(24-25): $2352-2364$.

[7] Păun, G.; Rozenberg, G.; Salomaa A. (ads.)(2010); The Oxford Handbook of Membrance Computing, Oxford Unversity Press, New York.

[8] Pan, L.Q.; Zeng, X.X. (2011); Small Universal Spiking Neural P Systems Working in Exhaustive Mode, IEEE Trans. on Nanobiosci., ISSN 1536-1241, 10(2): 99-105.

[9] Wang, T.; Wang, J.; Peng, H.; Wang, H. (2011); Knowledge Representation and Reasoning Based on FRSN P System, In: Proc of the 9th World Congress on Intelligent Control and Automation, pp. 849-854.

[10] Zhang, X.Y.; Luo, B.; Fang, X.Y.; Pan, L.Q. (2012); Sequential Spiking Neural P Systems with Exhaustive Use of Rules, BioSystems, ISSN 0303-2647, 108(1-3): 52-62.

[11] Francis G.C.; Henry N.A. (2012); On Structures and Behaviors of Spiking Neural P Systems and Petri Nets, Lecture Notes in Computer Science, ISSN 0302-9743, 7762: 145-160.

[12] Song, T.; Pan, L.Q.; Păun, Gh. (2013); Asynchronous Spiking Neural P Systems with Local Synchronization, Inform. Sciences, ISSN 0020-0255, 219: 197-207.

[13] Xiong, G.J.; Shi, D.Y.; Zhu, L.; Duan, X.Z. (2013); A New Approach to Fault Diagnosis of Power Systems Using Fuzzy Reasoning Spiking Neural P Systems, Math. Probl. Eng., ISSN 1024-123X, vol. 2013: Article ID 815352, 13 pages.

[14] Chen, W.H. (2011); Fault Section Estimation Using Fuzzy Matrix-based Reasoning Methods, IEEE Trans. on Power Deliver., ISSN 0885-8977, 26(1): 205-213.

[15] Chen, S M. (1996); A Fuzzy Reasoning Approach for Rule-based Systems Based on Fuzzy Logics, IEEE Trans. Syst., Man, Cybern., Syst., ISSN 1083-4427, 26(5): 769-778.

[16] Liu, H.C.; Liu, L.; Lin, Q.L.; Liu, N. (2013); Knowledge Acquisition and Representation Using Fuzzy Evidential Reasoning and Dynamic Adaptive Fuzzy Petri Nets, IEEE Trans. on Cybern., ISSN 1083-4419, 43(3): 1059-1072.

[17] Luo, X.; Kezunovic M. (2008); Implementing Fuzzy Reasoning Petri Nets for Fault Section Estimation, IEEE Trans. on Power Syst., ISSN 0885-8950, 23(2): 676-685.

[18] Chen, W.H.; Tsai, S.H.; Lin, H.I. (2011); Fault Section Estimation for Power Networks Using Logic Cause-effect Models, IEEE Trans. on Power Deliver., ISSN 0885-8977, 26(2): 963-971.

[19] The Matlab Website. http://www.mathworks.es/products/matlab/.

[20] Research Group on Natural Computing, University of Seville: The P-Lingua Website. http://www.p-lingua.org.

[21] Research Group on Natural Computing, University of Seville: The MeCoSim Website. http://www.p-lingua.org/mecosim. 